\title{
MN linked to improved prognosis in anti-GBM disease
}

Distinct antibody profiles and an improved prognosis are associated with the presence of membranous nephropathy $(\mathrm{MN})$ in patients with anti-GBM (glomerular basement membrane) antibody disease, according to findings just published by researchers at the Peking University, Beijing, China. "These data suggest that patients with combined disease can be classified as a unique subgroup of anti-GBM patients," the investigators write in their report.

The association of anti-GBM antibody disease with $\mathrm{MN}$ is widely recognized, with numerous individual cases documented; however, little is known about the immunological and clinical characteristics of these patients. The researchers, led by Zhao Cui, studied eight patients with combined disease, 30 patients with classic anti-GBM disease (without MN) and 20 patients with $\mathrm{MN}$ alone who presented to a single hospital. Patients with combined disease had milder kidney injury, as indicated by markedly reduced rates of oligouria and anuria, decreased gross haematuria, and lower serum levels of creatinine, compared to patients with anti-GBM disease alone. Renal outcomes following treatment were also better in the patients with combined disease; five of eight such patients remained dialysis-free, compared with just four of 30 patients with classic anti-GBM disease.

Patients with combined disease also had distinct anti-GBM antibody profiles. Sera from

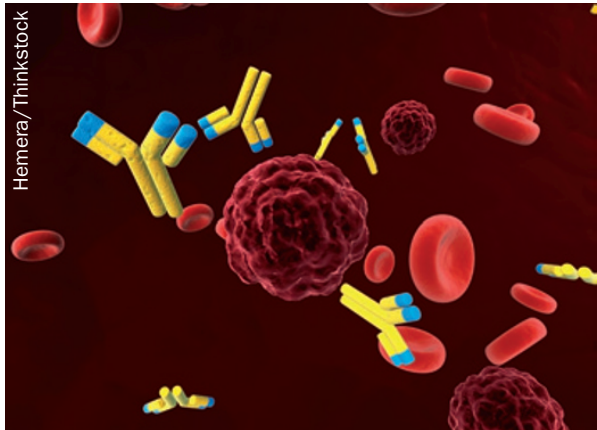

patients with combined disease had marked reactivity only to the a 3 chain of type IV collagen. By contrast, sera from patients with classic anti-GBM disease reacted to all five collagen $\alpha$ chains.

However, the researchers caution that this observation might be accounted for by decreased overall antibody reactivity, rather than a limited range of antibodies, in the patients with combined disease.

Interestingly, the patients with combined disease also had significantly lower titres of antibodies targeting the $\mathrm{E}_{\mathrm{B}}$ epitope of the $\alpha 3$ chain of type IV collagen than did those with classic antiGBM disease. The team's previous work linked these antibodies to an increased risk of end-stage renal disease. "These factors may contribute to the milder kidney damage and better renal prognosis in these patients," the authors' report concludes.

\section{David Holmes}

Original article Jia, X.-Y. et al. The clinical and immunological features of patients with combined anti-glomerular basement membrane disease and membranous nephropathy. Kidney Int. doi:10.1038/ki.2013.364 